\title{
AS METODOLOGIAS ATIVAS DE APRENDIZAGEM: UMA ANÁLISE DA PERCEPÇÃO DE FUTUROS PROFESSORES NO CURSO DE PEDAGOGIA
}

\author{
HUMBERTO VINÍCIO ALTINO FILHO ${ }^{1}$, ANDRÉIA ALMEIDA \\ MENDES ${ }^{2}$, CAMILA BRAGA CORRÊA ${ }^{3}$, LIDIANE HOTT DE FÚCIO \\ BORGES ${ }^{4}$, RITA DE CÁSSIA MARTINS DE OLIVEIRA VENTURA ${ }^{5}$, \\ REGINALDO ADRIANO DE SOUZA ${ }^{6}$
}

\footnotetext{
${ }^{1}$ Mestre em Educação Matemática pela UFOP, Especialista em Estatística pela FATESF, Licenciado em Matemática pela FACIG. Professor e Analista Educacional no Centro Universitário UNIFACIG. humbertovinicio@hotmail.com

${ }^{2}$ Doutora em Linguística pela Universidade Federal de Minas Gerais (UFMG), Coordenadora de Pesquisa e Extensão e professora do Centro Universitário UNIFACIG. andreialetras@ yahoo.com.br

${ }^{3}$ Mestra em Justiça Administrativa - PPGJA (UFF). Professora no Centro Universitário UNIFACIG. camilabragacorrea@gmail.com

4 Mestre em Engenharia e Ciência dos Materiais pela UENF. Professora e Coordenadora das Licenciaturas em Pedagogia, História e Matemática do Centro Universitário UNIFACIG. lidianehott@yahoo.com.br

${ }^{5}$ Doutora em Ciência da Informação pela Universidade Federal de Minas Gerais UFMG, pró-reitora e professora no Centro Universitário UNIFACIG dir.academica@ facig.edu.br

6 Mestre em Administração pela Faculdade de Estudos Administrativos de Minas Gerais FEAD, coordenador e professor no Centro Universitário UNIFACIG. reginaldoberbert@ hotmail.com
}

\section{RESUMO}

As metodologias ativas de aprendizagem encontram-se em foco nos estudos da educação na atualidade, uma vez que são meios potenciais de desenvolvimento de competências e habilidades demandadas para a sociedade atual e futura. Atentando-se para esse fato, é de suma importância incluir na formação de professores tais metodologias, com vistas a promoção da aprendizagem efetiva. Nesse sentido, a instituição de ensino superior brasileira, unidade de análise deste estudo, incluiu a disciplina "Metodologias Ativas de Aprendizagem" nas novas grades das licenciaturas. Este trabalho visa apresentar a percepção dos futuros professores, alunos do curso de Pedagogia, sobre as metodologias ativas vivenciadas na disciplina e sua transposição para a sala de aula da Educação Básica. A coleta de dados foi feita por meio de questionário de natureza quantitativa. As respostas do questionário abrangeram $90 \%$ dos alunos da turma que cursou a disciplina em questão, que, em sua maioria, tem de 18 a 20 anos e já esteve atuando em sala de aula. Os resultados apontam que $91 \%$ dos participantes encontram-se motivados para utilizar os métodos ativos em sua prática docente; afirmando que as aulas dialogadas, o Audiovisual e o Team Based Learning (TBL) são as metodologias mais adequadas para a Educação infantil e Anos Iniciais do Ensino Fundamental. Portanto, pode-se observar que, além de utilizar os métodos ativos em todo desenvolvimento do curso, a implantação de uma disciplina específica para tratar dessas metodologias prenuncia o emprego de metodologias ativas de 
aprendizagem pelos professores formados nesse formato, contribuindo de forma significativa para a transformação da sala de aula na Educação Básica.

Palavras-chave: Metodologias Ativas de Aprendizagem; Formação de Professores; Pedagogia; Educação Básica e Superior.

\title{
ACTIVE LEARNING METHODOLOGIES: AN ANALYSIS OF THE PERCEPTION OF FUTURE TEACHERS, IN THE PEDAGOGY COURSE
}

\begin{abstract}
Active learning methodologies are in focus in education studies currently as they are potential means of developing competencies and skills demanded by current and future society. Bearing in mind this fact, it is extremely important to include such methodologies in the teachers education, with a view to promoting effective learning. In this sense, the Brazilian higher education institution, the unit of analysis of this study, included the discipline "Active Learning Methodologies" in the new curriculum of teachers education. This work aims to present the perception of future teachers, students of the Pedagogy course, about the active methodologies experienced in the discipline and its transposition to the Basic Education classroom. Data collection was performed using a quantitative questionnaire. The responses to the questionnaire covered $90 \%$ of the students in the class that took the course in question, which, in its majority, is between 18 and 20 years old and has already been working in the classroom. The results show that $91 \%$ of the participants are motivated to use the active methods in their teaching practice; stating that the dialogued classes, Audiovisual and TBL (Team Based Learning) are the most appropriate methodologies for Pre-School and Elementary School. Therefore, it can be observed that, in addition to using the active methods in all course development, the implementation of a specific discipline to deal with these methodologies foreshadows the use of active learning methodologies by teachers formed in this format, contributing significantly to the transformation of the classroom into Basic Education.
\end{abstract}

Keywords: Active Learning Methodologies; Teachers Education; Pedagogy; Basic and Higher Education.

\section{INTRODUÇÃO}

O cenário da educação na contemporaneidade aponta para novos objetivos, baseados, majoritariamente, no desenvolvimento de competências e habilidades. Nesse sentido, surgem metodologias inovadoras com vistas a atender tais demandas educacionais, dentre essas, estão as metodologias ativas de aprendizagem.

As metodologias ativas são estratégias de aprendizagem centradas no estudante e na sua formação geral, integrando o processo de ensino e aprendizagem de conteúdos e 
o desenvolvimento de habilidades e competências, interligadas ao futuro contexto profissional e a atuação dos indivíduos na sociedade.

Sendo esses motes das metodologias ativas, há que se considerar de grande importância incluir essa temática nos cursos de formação de professores, uma vez que estes figurarão como agentes transformadores da educação hoje e no futuro.

Nessa esteira, esta pesquisa busca analisar a percepção de futuros professores da Educação Básica sobre aos métodos apresentados na disciplina "Metodologias Ativas de Aprendizagem", ministrada no curso de Pedagogia, de uma Instituição de Ensino Superior da Vertente Ocidental do Caparaó, em Minas Gerais, no Brasil.

\section{METODOLOGIAS ATIVAS E FORMAÇÃO DE PROFESSORES}

As Metodologias Ativas são estratégias de ensino, centradas no estudante, baseadas em "processos interativos de conhecimento, análise, estudos, pesquisas e decisões individuais ou coletivas, com a finalidade de encontrar soluções para um problema, um caso, ou construir e executar um projeto" (CECY; OLIVEIRA; COSTA, 2013, p. 17), oportunizando ao estudante atividades de alto desempenho cognitivo. Dessa forma, os métodos ativos configuram-se como importantes aliados no processo de ensino e aprendizagem.

A discussão sobre o contexto da educação e as novas metodologias perpassam os mais diversos âmbitos de formação profissional. No entanto, há de se concordar que tal debate é certamente interligado ao campo da formação de professores para os mais diversos níveis de ensino, uma vez que reconstrói a figura e os papéis do docente.

Numa ambiência, cuja discussão em voga é o ensinar, ato distintivo da profissão docente, apresentando-se "nesta leitura, essencialmente como a especialidade de fazer aprender alguma coisa (a que chamamos currículo, seja de que natureza for aquilo que se quer ver aprendido) a alguém" (ROLDÃO, 2007, p. 95), colocar em tela questões relacionadas ao modo de promover situações de ampla aprendizagem, com metodologias adequadas ao contexto sócio-histórico-cultural, é consentaneamente válido.

Estando a figura do aluno cada vez mais numa posição de protagonista do processo de aprendizagem, para o professor, surgem novos desafios como organizar e 
dirigir situações de aprendizagem; administrar a progressão das aprendizagens; conceber e fazer evoluir os dispositivos de diferenciação; envolver os alunos em sua aprendizagem e em seu trabalho; trabalhar em equipe; utilizar novas tecnologias; administrar sua própria formação continuada (PERRENOUD, 2000).

As Metodologias Ativas de aprendizagem viabilizam a discussão de todo esse cenário construído pelas transformações da sociedade, uma vez que contemplam em suas características o desenvolvimento das competências necessárias para o séc. XXI, como a resolução de problemas complexos, o pensamento crítico, a capacidade de julgamento e tomada de decisão, a flexibilidade cognitiva (WORLD ECONOMIC FORUM, 2016).

Dessa forma, espera-se que o professor seja capaz de mobilizar os saberes do conhecimento do conteúdo, do conhecimento curricular e do conhecimento pedagógico do conteúdo com foco no processo de ensino e aprendizado benfazejo (SHULMAN, 1986).

Atentando-se para essa discussão, a formação de professores tem passado por mudanças incluindo em seu currículo disciplinas relacionadas aos procedimentos educacionais baseados na aprendizagem ativa e empregando métodos ativos nas disciplinas relacionadas aos conhecimentos disciplinares, assegurando, dessa maneira, que mesmo as aulas que tratam dos conteúdos exclusivos da área, tenham aporte nos princípios didáticos que se objetiva desenvolver.

\section{METODOLOGIA}

Trata-se de uma pesquisa aplicada e explicativa, de caráter quantitativo. Para a coleta de dados, optamos por utilizar um Survey, disponibilizado aos alunos da disciplina de forma eletrônica, uma vez que as informações sobre o contato com a disciplina foram coletadas diretamente com o grupo de interesse (SILVELRA; CÓRDOVA, 2009). Os participantes da pesquisa foram os discentes do curso de Licenciatura em Pedagogia que haviam cursado a disciplina "Metodologias Ativas de Aprendizagem" durante o $3^{\circ}$ período no primeiro semestre de 2019 . No total, a turma era composta de 12 estudantes, dos quais 11 responderam ao questionário deste estudo. 


\section{RESULTADOS E DISCUSSÃO}

Nesta seção, apresentam-se os resultados obtidos pelas respostas dos estudantes no questionário. Inicialmente, podemos ressaltar que $90 \%$ do total de alunos da disciplina responderam à pesquisa, totalizando 11 alunos.

De acordo com o gráfico abaixo (ver Gráfico 1), nota-se que a maior parte dos alunos dessa turma tem idade entre 18 e 20 anos, apresentando-se, portanto, como um grupo jovem de estudantes. Apenas 1 aluno tem entre 31 e 40 anos e outros 2, tem idade de 21 a 30 anos

Gráfico 1 - Idade dos Estudantes

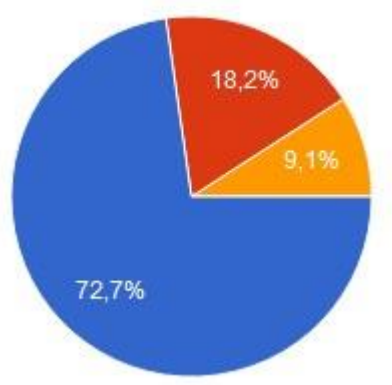

18 a 20 anos

Fonte: Dados da Pesquisa.

Questionados sobre contatos práticos com a experiência docente, todos alunos informaram que já atuaram em sala de aula, seja pelo Programa Institucional de Bolsas de Iniciação à Docência (PIBID/CAPES), por substituição dos professores titulares ou por estágios supervisionados.

O gráfico apresentado abaixo (ver Gráfico 2) mostra que 90,9\% da turma encontra-se altamente motivada para incorporar as metodologias ativas de aprendizagem em sua prática docente. Sendo que apenas 1 aluno informou um grau de motivação mediano para a utilização dos métodos ativos em sua sala de aula. 
Gráfico 2 - Grau de motivação dos estudantes para a utilização de metodologias ativas em sua prática docente

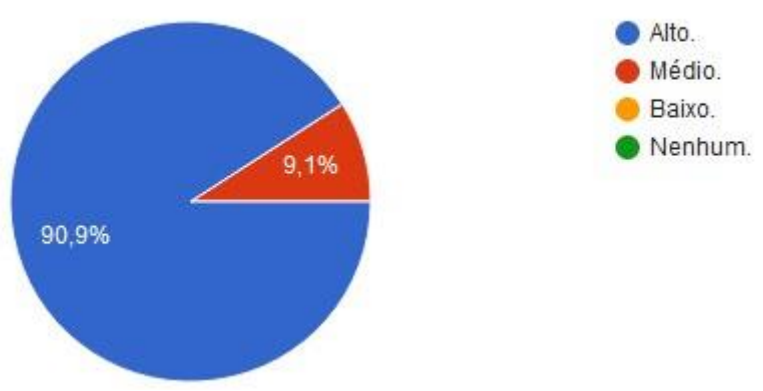

Fonte: Dados da Pesquisa.

Pelos dois gráficos seguintes (ver Gráfico 3 e Gráfico 4), investiga-se a avaliação dos estudantes sobre a adequação das mais diversas metodologias de ensino e aprendizagem aos níveis de ensino para os quais estarão habilitados a lecionar quando egressarem do curso.

Gráfico 3 - Métodos de ensino mais adequados à Educação Infantil de acordo com os estudantes

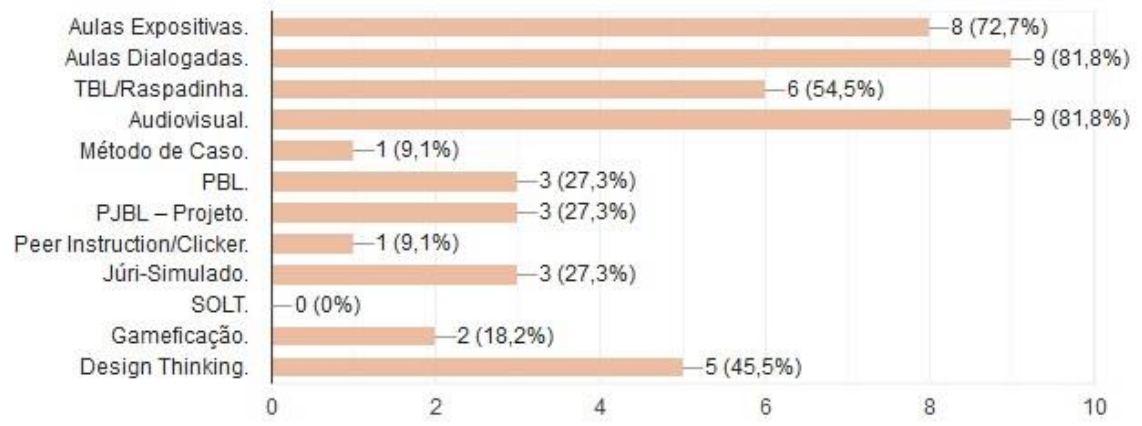

Fonte: Dados da Pesquisa. 
Gráfico 4 - Métodos de ensino mais adequados aos Anos Iniciais do Ensino Fundamental, de acordo com os estudantes

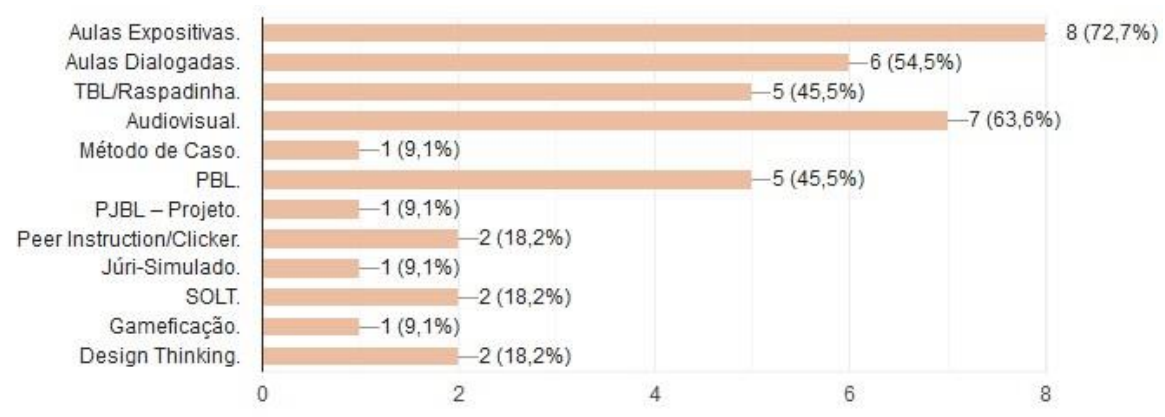

Fonte: Dados da Pesquisa.

Observando os gráficos, pode-se notar que as aulas expositivas e dialogadas, o Audiovisual e o TBL (Team Based Learning) são as metodologias mais adequadas tanto para a Educação infantil, quanto para os Anos Iniciais do Ensino Fundamental, segundo os discentes.

De acordo com o Gráfico 5, abaixo, nota-se que a maior parte dos alunos do curso de Pedagogia da instituição não haviam tido outro contato com oportunidades formativas relacionadas às Metodologias Ativas de Aprendizagem, a não ser a própria disciplina.

Gráfico 5 - Contato com outros cursos de Metodologias Ativas de Aprendizagem

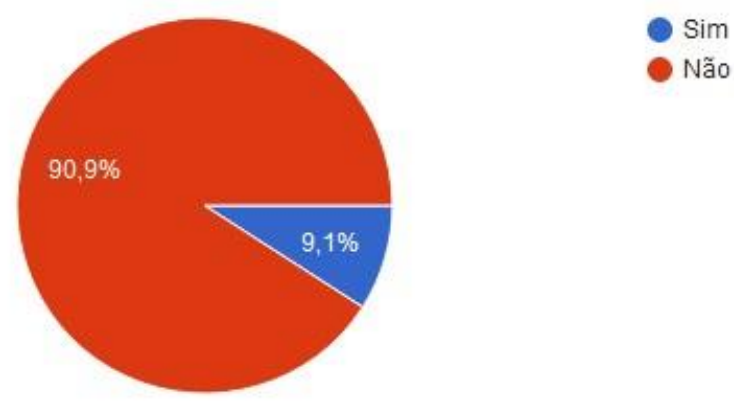

Fonte: Dados da Pesquisa.

Os dois gráficos seguintes (ver Gráfico 6 e Gráfico 7) apresentam as respostas dos alunos acerca dos obstáculos para o emprego de Metodologias Ativas em escolas da rede pública e privada. 
Gráfico 6 - Dificuldades para aplicação do método de ensino ativo em escolas públicas.

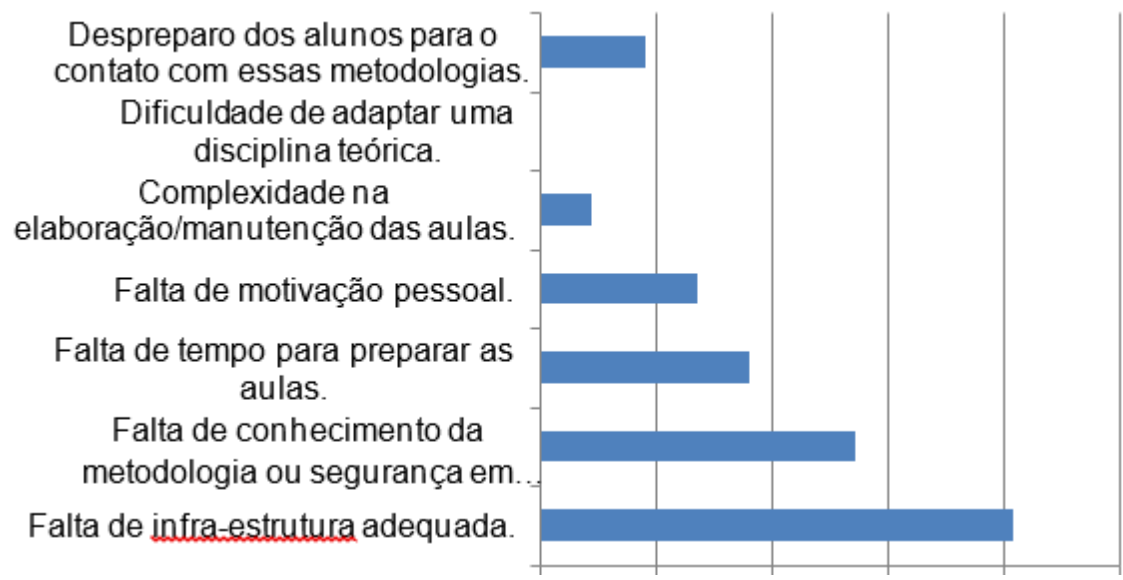

Fonte: Dados da Pesquisa.

Gráfico 7 - Dificuldades para aplicação do método de ensino ativo em escolas públicas.

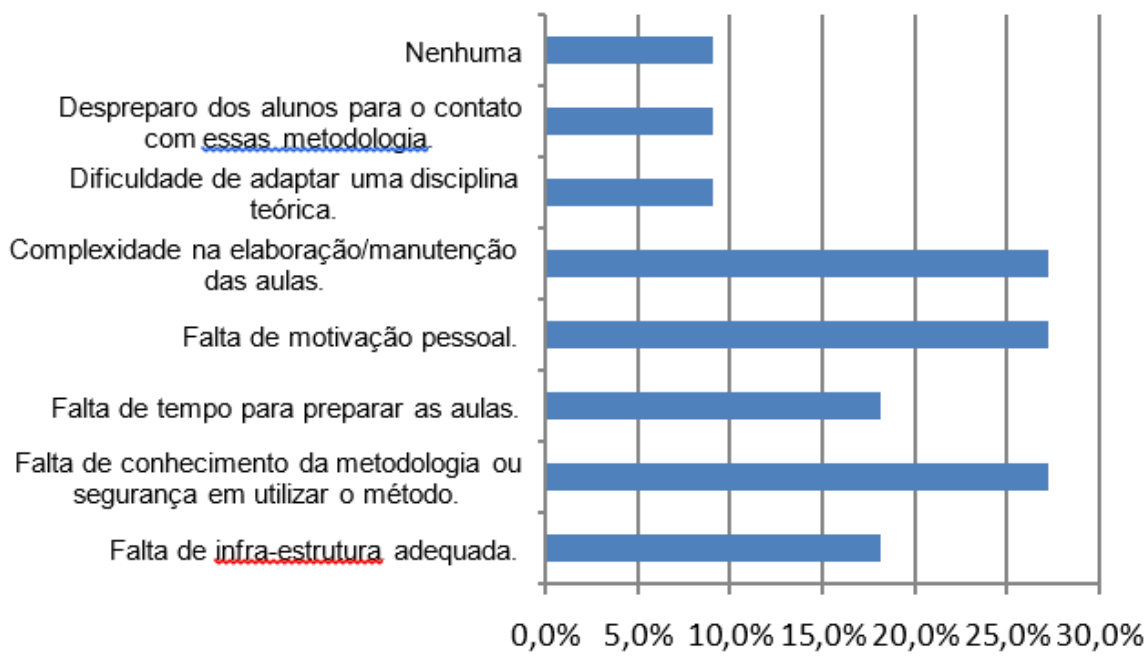

Fonte: Dados da Pesquisa

Pela análise dos gráficos, observa-se que, no caso das escolas públicas, a infraestrutura foi apontada como um grande obstáculo para a utilização das metodologias ativas, sendo apontada por mais de $80 \%$ dos respondentes. Nas escolas particulares, todos os fatores foram citados com menor frequência, apresentando uma distribuição maior dos resultados. A falta de conhecimento da metodologia ou segurança em utilizar o método foi indicada como elemento dificultador em ambos os casos.

Por fim, os estudantes foram convidados a estabelecer uma comparação das metodologias ativas em relação ao método tradicionais. Dos 11 alunos participantes da 
pesquisa, nove responderam a esse questionamento. As respostas seguem agrupadas na tabela abaixo.

Tabela 1 - As Metodologias Ativas em relação ao método tradicional

\begin{tabular}{|c|c|}
\hline \multirow{2}{*}{$\begin{array}{l}\text { Relação com } \\
\text { a Inovação }\end{array}$} & Inovador e eficaz. \\
\hline & $\begin{array}{l}\text { Uma inovação ao método tradicional, metodologia ativa é uma ótima forma } \\
\text { de se ensinar. }\end{array}$ \\
\hline \multirow{5}{*}{$\begin{array}{l}\text { Destaque } \\
\text { para a Pers- } \\
\text { pectiva do } \\
\text { Aluno }\end{array}$} & Um ótimo instrumento para que o aluno aprenda de fato \\
\hline & Muito bom para o aprendizado e desenvolvimento dos alunos. \\
\hline & Fazer com que o aluno tenha mais interesse para estudar e aprender. \\
\hline & $\begin{array}{l}\text { Metodologia ativa é a melhor forma de se trabalhar com crianças, despertar a } \\
\text { curiosidade da criança e fundamental para que ela aprenda e as metodologias } \\
\text { ativas são excelentes por trazer a criança como centro do aprendizado. }\end{array}$ \\
\hline & Ensino inclusivo. \\
\hline \multirow{2}{*}{$\begin{array}{l}\text { Opção } \\
\text { Metodológica }\end{array}$} & $\begin{array}{l}\text { Tem seu lugar, pois a forma como se dá é interessante e desperta a } \\
\text { curiosidade do aluno, pelo novo. }\end{array}$ \\
\hline & $\begin{array}{l}\text { Importantíssima pois o desenvolvimento dos alunos é alto considerado ha } \\
\text { outras praticas de desenvolvimento dos alunos. }\end{array}$ \\
\hline
\end{tabular}

Fonte: dados da pesquisa.

Pelas respostas, vê-se que as impressões dos estudantes sobre as metodologias ativas são positivas e há uma concentração na percepção relacionada ao foco no aprendizado dos alunos.

Com base nos resultados apresentados, pode-se analisar a percepção dos estudantes de Pedagogia sobre as Metodologias Ativas de Aprendizagem, principalmente, tratando-se dos métodos desenvolvidos na disciplina cuja temática era essa.

Pode-se ver que os estudantes, em sua maioria, apresentaram-se inclinados a uma boa relação com os métodos ativos e sua utilização em sala de aula. Por se tratar de uma turma bem jovem, pode-se dizer que os próprios alunos estão imersos no contexto de transformação vivido pelos cenários educacionais e podem mobilizar esses conhecimentos para os desenvolvimentos das habilidades necessárias para o professor 
do século XXI (PERRENOUD, 2000), por meio das atividades baseadas nas metodologias ativas que - através de processos de resolução de problemas, análises de casos, construção de projetos etc. - desenvolvem e/ou potencializam tais habilidades.

A transposição dessas atividades para a sala de aula da Educação Básica também foi indicada pelos futuros professores com alto grau de motivação, mesmo apresentando como dificuldades as questões infraestruturais, de domínio das metodologias, tempo para preparação, dentre outros.

Nota-se que as aulas dialogadas e o ensino Audiovisual foram apontados como métodos adequados para a aplicação no ensino básico. Outra metodologia citada pelos alunos como possibilidade de utilização para a Educação Infantil e Anos Iniciais do Ensino Fundamental, foi a Team Based Learning (TBL), que tem como foco a aprendizagem colaborativa, desenvolvendo habilidades de comunicação e interação entre pares (BOLELLA et al., 2014). Além dessas, outras metodologias como a Problem Based Learning e o Design Thinking também foram citadas em menor escala. As aulas expositivas também são consideradas pelos discentes como estratégias adequadas ao ensino, o que é previsível, uma vez que os estudantes vêm de uma base educacional ainda pautada no método tradicional. Há de se considerar também que a ideia da implantação de novas metodologias de aprendizagem, não é banir e/ou condenar as aulas expositivas e, sim, utilizá-las em situações bem planejadas e explorando seus potenciais.

No que se diz da comparação entre as Metodologias Ativas e o método tradicional, os estudantes reconheceram, em sua maioria, a importância e a potencialidade dos métodos ativos em priorizar o processo de aprendizado centrado no estudante, notando-se também as características inovadoras dessas metodologias, que podem ser conjugadas e/ou intercaladas com outras abordagens metodológicas de ensino e aprendizagem, possibilitando uma reflexão e interligação sobre o conhecimento pedagógico e o conhecimento pedagógico do conteúdo (SHULMAN, 1986).

\section{CONSIDERAÇÕES FINAIS}

Este estudo teve como objetivo analisar a percepção de futuros professores da Educação Básica, mais especificamente, alunos do curso de Pedagogia de uma 
instituição de ensino superior, que oferece uma disciplina específica para tratar das Metodologias Ativas de Aprendizagem sobre tais métodos.

Com base nos resultados e na interface com as pesquisas educacionais apresentadas, pode-se considerar que os professores se apresentaram motivados para a utilização de métodos ativos em sua prática docente, promovendo assim, a transposição das metodologias vivenciadas e discutidas em sua formação superior para a Educação Básica.

Dessa forma, pode-se concluir que a instrumentalização de Metodologias Ativas e a discussão desses métodos no espaço de formação de professores é de grande valia, tanto para o desenvolvimento das competências necessárias ao professor da atualidade quanto para a transformação e inovação nas salas de aula da Educação Básica.

\section{REFERÊNCIAS}

BOLLELA, Valdes Roberto et al. Aprendizagem baseada em equipes: da teoria à prática. Medicina (Ribeirao Preto Online), v. 47, n. 3, p. 293-300, 2014.

CECY, Carlos; OLIVEIRA, Geraldo Alécio de; COSTA, Eula Maria de Melo Barcelos. Metodologias Ativas: aplicações e vivências em Educação Farmacêutica. Brasília: Abenfarbio, 2013.

PERRENOUD, Philippe.: Dez Novas Competências para Ensinar. Artmed: Porto Alegre, 2000.

ROLDÃO, Maria do Céu. Função docente: natureza e construção do conhecimento profissional. Revista Brasileira de Educação, v. 12, n. 34, p. 94-103, 2007.

SHULMAN, Lee S. Those who understand: Knowledge growth in teaching. Educational researcher, v. 15, n. 2, p. 4-14, 1986.

SILVEIRA, Denise Tolfo,; CÓRDOVA, Fernanda Peixoto. A Pesquisa Científica. In: GERHARDT, Tatiana Engel; SILVEIRA, Denise Tolfo (Orgs). Métodos de Pesquisa. Porto Alegre, RS: Editora da UFRGS, 2009, p. 31-42.

WORLD ECONOMIC FORUM. Fórum Econômico Mundial. Disponível em: http://www3.weforum.org/docs/WEF_New_Vision_for_Education.pdf. Acesso em: 20 mar. 2020.. 